\title{
P5: Entwicklung eines Objective Structured Clinical Examination (OSCE) für das erste Ausbildungshalbjahr in der Gesundheits- und Kranken-/Kinderkrankenpflege
}

\author{
Marion Fischer $\cdot$ Luisa Weidauer
}

Online publiziert: 23. Oktober 2013

(C) Springer-Verlag Wien 2013

Einleitung/Fragestellung: Seit der Novellierung des Krankenpflegegesetzes stehen berufliche Handlungskompetenzen im Fokus der Ausbildung. Kompetenzbasierte Curricula gelten als effektive Ausbildungsform. Als zugehörige mündlich/praktische Prüfungsform hat sich das für das Medizinstudium entwickelte OSCE bewährt, ein Parcours, an dem Aufgaben beruflichen Handelns bearbeitet und mittels standardisierter Bewertungsmaßstäbe beurteilt werden.

Für die Einschätzung der pflegerischen Handlungskompetenz im ersten Ausbildungshalbjahr haben die Autoren ein OSCE entwickelt, das bisherige Prüfungen ersetzt. Es ermöglicht den Auszubildenden eine hohe Eigenaktivität, Praxisnähe und das Üben berufsrelevanter Fertigkeiten (Skills).

Methodik: Nach Festlegung des Prüfungsanlasses werden bedeutsame Tätigkeitsfelder, wie z. B. „Haut/Körper pflegen“ oder „Hygienisch arbeiten“ extrahiert, um daraus konkrete Handlungen für OSCE-Stationen festzulegen. Beispiele sind u. a. „Eine Nabelpflege durchführen“, „Den Hautzustand beurteilen“ oder „Die hygienische Händedesinfektion durchführen“. Die im Blueprint festgelegten Handlungskompetenzen werden genutzt, um die Ausgestal- tung der Prüfungssituation realitätsnah (Raumgestaltung, Rolle des Schauspielpatienten) zu gestalten und um eine Checkliste zu erstellen, die eine objektive Bewertung des Auszubildenden durch einen lediglich beobachtenden Prüfer gewährleistet.

Ergebnisse: Das Poster präsentiert ein zwölfschrittiges OSCE, das die Möglichkeit bietet, klinische Kompetenzen der Auszubildenden zu prüfen. Zur Verdeutlichung des Procedere wird die Beispielstation „Eine hygienische Händedesinfektion durchführen“ ausformuliert. Sowohl die Relevanz der hygienischen Händedesinfektion, als auch die dazugehörigen beruflichen Handlungskompetenzen werden benannt; ebenso die Beschreibung der Situation und die Formulierung der konkreten Aufgabenstellung. AbschlieBend wird die Prüfungscheckliste dargestellt.

Diskussion/Schlussfolgerung: Der Einsatz eines OSCE kann das moderne Ausbildungsverständnis bereichern und seinen festen Platz in der Gesundheits- und Kranken-/Kinderkrankenpflegeausbildung finden. Trotzdem ist das OSCE gezielt einzusetzen, da erhebliche zeitliche, personelle und räumliche Ressourcen benötigt werden.

M. Fischer $(\triangle) \cdot$ L. Weidauer

Fachhochschule Münster, Fachbereich Pflege und Gesundheit,

Münster, Deutschland

E-Mail: Marion.Fischer@fh-muenster.de 